\title{
Comparative Study of Different Wavelet Transforms in Fusion of Multimodal Medical Images
}

\author{
R. E. Masumdar \\ Intern at Maven Silicon \\ Softech Pvt Ltd, \\ \#633, Govinda Reddy Layout, \\ Arekere Main Road, Bangalore
}

\author{
R. G. Karandikar \\ Dept of Electronics \& Telecommunication, \\ KJ Somaiya College of Engineering, \\ Vidyavihar, Mumbai-400077
}

\begin{abstract}
This paper presents a comparative study of image fusion of MRI and CT images using various wavelet transforms. The fusion of the images is done by implementing a multiresolution decomposition method with the help of various wavelets. Entropy, PSNR and MSE are the parameters that are used as performance metrics of the fusion done using various wavelets. The MRI and CT images are then fused using the select maximum fusion rule, since studies have shown that select maximum rule provides the best result. The final fused image is examined using the various performance metrics to evaluate which wavelet gives the best result.
\end{abstract}

\section{Keywords}

Computed Tomography, Magnetic Resonance Imaging, Wavelet Transform, Haar Transform, Daubechies Transform, Symlet Transform, Image Fusion Vanishing Moments, Multiresolution Decomposition, Image Fusion, Quadrature Mirror Filter, Order, Filter Banks, Mean Squarred Error, Peak Signal to Noise Ratio, Entropy

\section{INTRODUCTION}

In Modern medicine, a number of medical imaging technologies have been developed, each with their own advantages and disadvantages. These techniques are used to acquire information about an affected area, usually in pairs of two different images having complementary properties. These images which when viewed simultaneously by doing fusion can present us with a good diagnosis rather than viewing them separately. CT imaging provides the information about brain/skeletal injuries. While MRI provides details of soft tissue and anatomic structures of gray and white matter present in brain. Both these modalities cannot carry all the complimentary and relevant information in single image. But fused image of these two provides us the entire information in a single image, facilitating more precise diagnosis and a more accurate treatment.

In any classical Image Fusion task, an image undergoes Image Registration and fusion of relevant features from source images. Registration of image deals with the corrections to be made in spatial misalignments while feature extraction mainly concerns with identification and selection of features with a focus on relevance of features for a given clinical condition which is of three categories namely Pixel level fusion[1], Feature level fusion[1], Decision level fusion[1].

Image Fusion further takes an approach into two different ways, known commonly as Spatial domain and Transform Domain [2]. Spatial Domain method deals directly with the pixels of an image. The pixels of source images undergo manipulations in them to produce a final fused image. Some of the methods that fall into the spatial domain fusion category are Brovey Transform[3], PCA - Principal Component Analysis[4], Intensity Hue Transformation[5] and many more to name.

The spatial distorsion in an image being the downside of spatial domain, which makes transform domain fusion methods more advantageous..Out of the two types of transform domain methods the wavelet transform method is chosen more over pyramid transform due to its property of blocking effect in fused image[6].

Pyramid and Wavelet Transforms are two of the few commonly used transform domain methods used for image fusion. Pyramid transform methods are further categorized into Laplacian transform, Gradient transform, etc. Similarly there are numerous different methods that fall under the wavelet transform category, but it is wavelet transform that is chosen more over pyramid transform, the reason being the blocking effect pyramid transform methods induce in fused images. A more detailed explanation of blocking effect can be seen in reference [6].

Features of an image are characterized by a different physical structure at a different resolution. Images with larger structures correspond to details which comfortably provide image context by simply having a glimpse, while images with finer resolution gives a detail visual information about small objects. From Medical Imaging point of view, multimodal images generally emphasize different anatomical structures in either a poor or a good spatial resolution, but usually appear in complimentary image form. Thus, analyzing images at different resolution becomes a mandatory condition. While, analyzing these images though, the interpretation of the scene should be invariant with the modifications in the scale. Hence, multiresolution decomposition[6]. Besides these primary advantages the most important and distinguishing character of multiresolution decomposition is that it provides information on the magnitude of rapid contrast changes in an image. Since, human eyes are sensitive to local changes in contrast, it serves to act as information provision scheme for an observer[7].

Wavelet transform and pyramid transforms both can be used for fusion of images by implementing multiresolution decomposition algorithm. From among the existing algorithms use of DWT (Discrete Wavelet Transform) is more generic and finds application in many other image processing practices. A reason for its common use is its merit of preserving different frequency information in stable form and allowing good localization both in time and spatial frequency domain.

Although DWT finds a common application in medical image fusion, the extent to which the various wavelets bases can serve in fusion of images efficiently is still a hot topic for 
research. This paper draws a comparative study of different wavelet bases and their effectiveness in medical image fusion applications using the transform like Symlet, Daubechies, and Haar in fusion of medical images. The quality of performance of fusion is compared with the help of measures like PSNR, MSE and Entropy. Rest of the paper will give an insight into different wavelet bases. A brief discussion of image fusion method based on multi-resolution decomposition will be done. Conclusively presenting the comparative results presented in a tabular form followed by conclusion and appropriate references.

\section{DISCRETE WAVELET TRANSFORM}

Wavelet Transform can be viewed as a function that is used to represent another function onto which the operations of scaling and dilation are performed. Wavelet Transform can be expressed as an integral product of a signal to be analyzed with a scaled and dilated version of another signal (commonly referred to as wavelet function)

$$
W T=a^{-\frac{1}{2}} \int x(t) \psi^{*}\left(\frac{t-\tau}{a}\right)
$$

The other signal is named as Wavelet function. A general expression for Wavelet function can be given as follows:

$$
\psi_{(a, b)}(t)=|a|^{-\frac{1}{2}} \psi^{*}((t-b) / a)
$$

Mother wavelet can be thought of as a function responsible for representation by superposition of its various scaled and dilated versions of any arbitrary signal. It also possess a peculiar property that allows us to reconstruct the original signal which has undergone multiresolution wavelet decomposition. Wavelet transform of any function $f(x) \in$ $L^{2}(R)$ is stably invertible provided that the mother wavelet also is an element of $L^{2}(R)$. These wavelet functions were first introduced by Grossman and Morlet [8]. But, the property that the scaling and dilations of them can be used as expansion of functions $\epsilon L^{2}(R)$ was first brought to notice by Meyer [9]. Meyer said that the dilated and scaled versions of the mother wavelet forms an orthonormal basis for all the square integrable functions.

As was described earlier (in the introduction) the details of an image at any resolution can be better obtained by taking the difference between the approximations of two successive resolutions. Thus, to decompose an image into multiple resolutions wavelets are used[10]. The decomposition of an image is generally done in steps of two. Thus, the same applies for the variables of scaling and dilation in a wavelet function, resulting in expression of a term coined as a daughter function.

$$
\psi(x)=\sqrt{2^{j}} \psi\left(2^{j} x-k\right)
$$

here $a=2^{j}$ and $b=k 2^{j}$

A signal can be considered as a vector space given by $V_{2}^{j} \mid V_{2}^{j}$ $\subset L^{2}(R)$. The approximations of an image at resolution $2^{j}$ can be obtained by operating an orthogonal projection onto the vector space. Later, to avoid the inconveniency caused by the discontinuity and the absence of smoothness in the function of the vector space a unique function called as scaling function [11] was introduced. The dilation and scaling of which makes them orthonormal basis of the vector space $V_{2^{j}}$. In the very similar manner, a wavelet function forms an orthonormal basis for a vector space $O_{2}$ which is orthogonal to the vector space $V_{2^{j}}$. This manner of calculating coefficients makes the multiresolution representation an efficient pyramidal algorithm. A rather generalized set of expressions for the scaling and wavelet coefficients was given by S. G. Mallat[10] as follows:

$$
\begin{aligned}
& D_{m, n}=\sum g_{2 n-k} A_{m+1, k} \\
& A_{m, n}=\sum h_{2 n-k} A_{m+1, n}
\end{aligned}
$$

In the above manner coarser approximations as well as detail approximations can be estimated recursively by filtering followed by down-sampling operation (by a scale of two).

Finite length FIR filters can be used for decomposition sake. These filters are entirely capable to decompose the original signal/image. But, they simultaneously exhibit a drawback of generating a non-linear phase, when used for reconstruction of the image/signal reason being their anti-symmetric nature. To overcome this limitation, Mallat gave a relation between the filters, referred to as Quadrature Mirror Filters[10]. The relationship between their coefficients can be given as:

$$
g(n)=(-1)^{1-n} h(1-n)
$$

In many a cases, it becomes a task to choose a wavelet and scaling functions such that they meet the condition of mirrored impulse response filters, while simultaneously abiding the notion of orthogonality. Thus, for such cases, a group of wavelets known as Bi-orthogonal wavelets are used [11].

The above derivation works well in accord with 1-D signals, but it also serves well when used as an extension for 2-D signals or images. When it comes to image signals though a vector space $V_{2^{j}} \mid V_{2^{j}} \subset L^{2}\left(R^{2}\right)$ can be considered to be a tensor product of vector subspaces each belonging to $L^{2}(R)$ [10].

$$
V_{2^{j}}=V_{2^{j}}^{1} \otimes V_{2^{j}}^{1}
$$

A necessary condition though for $V_{2^{j}}$ to be a multi-resolution approximations of $L^{2}\left(R^{2}\right)$ is that the vector subspaces $V_{2^{j}}^{1}$ are multiresolution approximations of $L^{2}(R)$. Depending on the above statement, wavelet and scaling functions for 2-D can be given as follows[11].

$$
\begin{aligned}
\phi(m, n) & =\phi(m) \phi(n) \\
\psi^{1}(m, n) & =\phi(m) \psi(n) \\
\psi^{2}(m, n) & =\psi(m) \phi(n) \\
\psi^{3}(m, n) & =\psi(m) \psi(n)
\end{aligned}
$$

In a very similar fashion as that of 1-D wavelet analysis, the 2-D wavelet analysis too consist of filtering followed by down-sampling. This is illustrated in figure. no. 1.

A typical J-level decomposition of an image follows a filtering along the rows to separate the lower frequencies and the higher frequencies, the impulse response of the filters of which are dependent on wavelet and scaling coefficients following a orthogonality condition. 


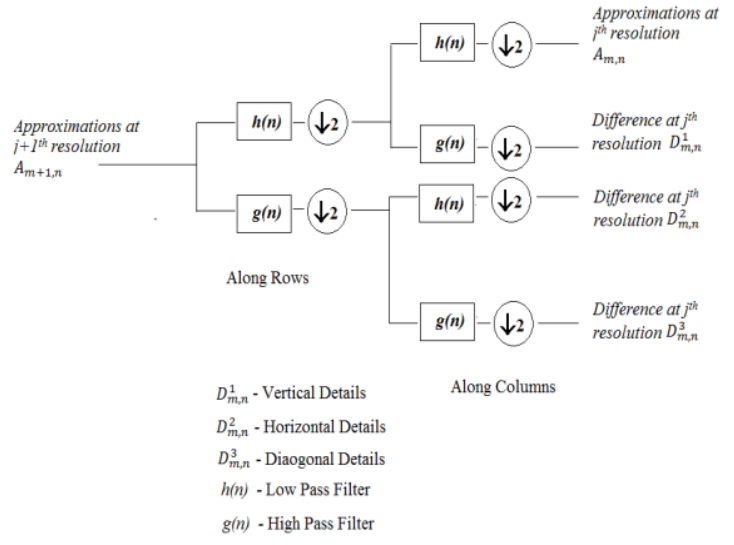

Fig 1: A single level Image Decomposition process. A low pass and high pass filtering followed by down sampling along rows and then columns.

Later filtering and down-sampling of the filtered Low and High bands along the row are followed by one along the columns giving rise to an approximation LL band, vertical details given by LH band, HL band for horizontal details. If again, one chooses to decompose an image by an additional level-J, the approximations obtained in LL band are decomposed to form four frequency bands. This further proceeds in a similar fashion, seperating the approximations and different detail signals.

As the main agenda of this paper is to present a comparative study of different wavelet bases that can be used for fusion of medical images, an attempt has been made to draw a qualitative comparison of fusion using Symlet, Daubechies and Haar wavelets. The next section gives a brief description of these wavelets.

\subsection{Haar Wavelet}

The Haar Functions are known since a hungarian mathematician named Alfred Haar [12] first discovered them. Since then a lot of modifications and changes have been made in the definition and generalizations [13][14][15][16]. It serves in many of the image processing applications. It has been used in coding of an image, feature extraction and many more promising techniques.

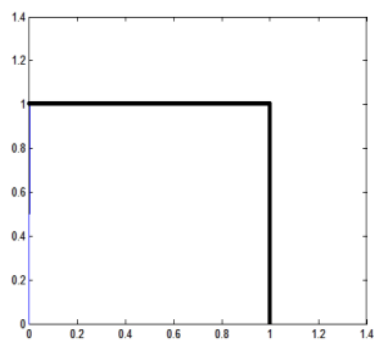

(a)

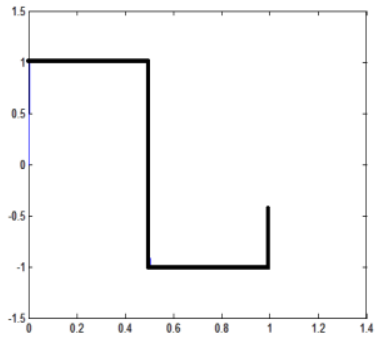

(b)
Fig 2: Haar Functions. Scaling Function on the left and Wavelet function on the right.

Haar can be defined as an orthogonal system that takes values generally from the set of $\left\{0,2^{j}: j \in N\right\}$, with its domain generalized as $L^{p}[0,1], p \in[0, \infty]$. The graphical representation of the Haar functions is as shown in following figure. no. 2 .

\subsection{Daubechies Wavelet}

These wavelets were first introduced by Ingrid Daubechies. The definition of Daubechies wavelet transform resemble that of Haar wavelet transform definition, the only manner in which they differ is the dissimilarity of definitions of the their scaling and wavelet signals.
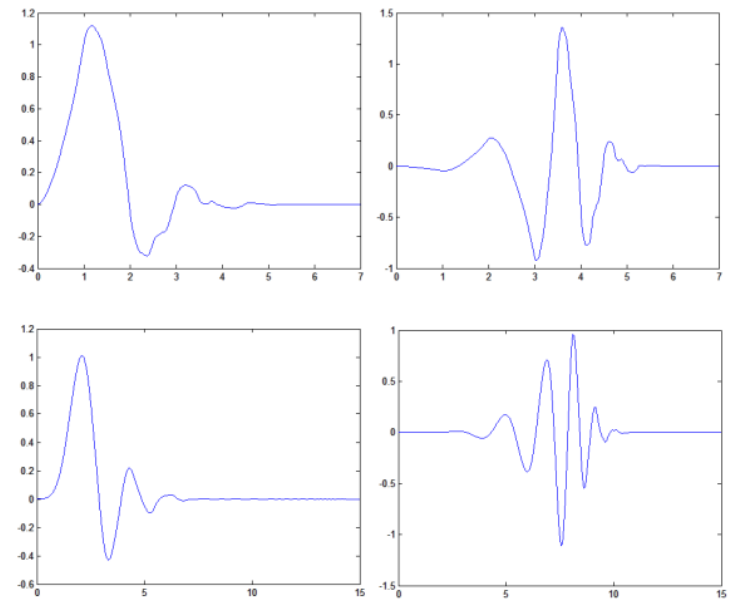

Fig 3: Daubechies wavelets with different orders.

These wavelet types also has balanced frequency response but exude non-linearity. A massive improvement can be seen in the results when Daubechies wavelet transforms are used, since the scaling and wavelet signals have relatively longer support. As compared to scaling and wavelet signals of Haar, the signals corresponding to Daubechies wavelet transform produce approximations and differences using a few more values[9].

\subsection{Symlet Wavelet}

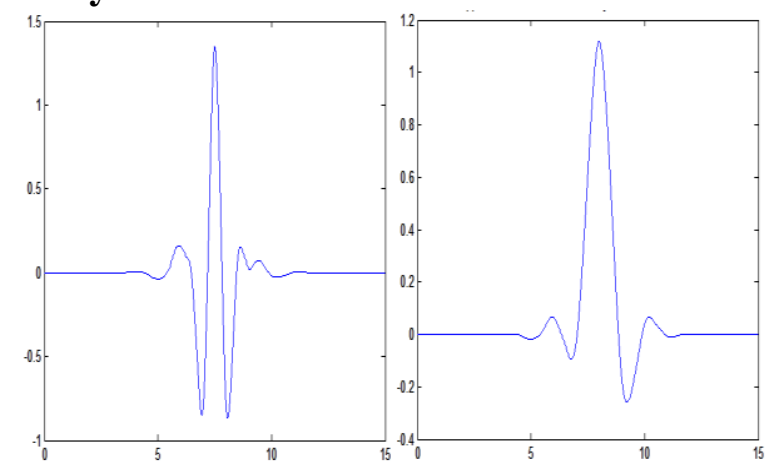

Fig 4: Symlet Scaling Function

Symlet Wavelets are very much similar to the Daubechies wavelets except the only difference being the vanishing moments of the wavelets function. Thus the wavelet coefficients differ than that of the daubechies'.

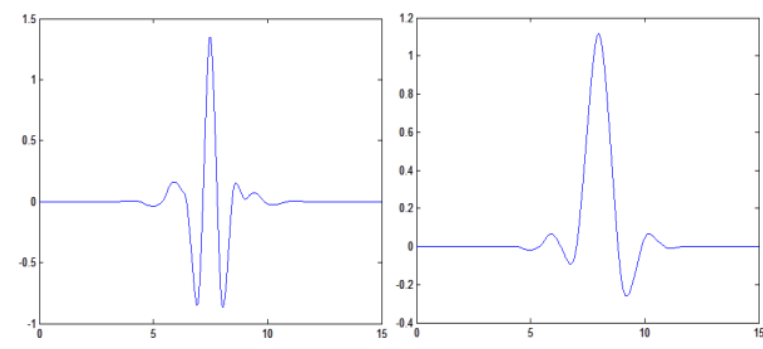

Fig 5: Symlet Scaling Function 


\section{IMAGE FUSION SCHEME}

It is necessary that the two images that are supposed to undergo the fusion are first registered. The registration of the images render the pixels of each image aligned, making it efficient to carry out the coefficient merging process. This is followed by wavelet transform calculation, giving rise to different frequency bands as described earlier. Each pixel in each of the frequency band correspond to pixel of the original image. The decomposition of an image into different frequency provides us with an option of having to choose from among four different bands corresponding to distinguishing features of an image. While the LL band provides with coarse features of an image, the LH, HL and $\mathrm{HH}$ band give a detail blueprint of vertical, horizontal and diagonal changes in intensity of pixels. The two images to be fused can be at different decomposition levels while being fused but it is uncompromisingly necessary to have the images at the same resolution level.

The image fusion scheme is a method that addresses the issue of representation of fusion of images. For any image the activity level reflects the local energy in the space spanned by the term in the expansion corresponding to a particular coefficient. Based on these assumptions there are three different methods categorized for computing activity level, better known as coefficient-based, region-based, and windowbased. Depending on the application to be served, the choice is made from among the above three methods of activity measurement[17]. The representation of an multi-scale decomposition coefficient can be well generalized as has been done in ref [17]. The multi-scale decomposition representation of an image is denoted as $D_{I}$ while the activity level is represented by $A_{I}$. For the images $\mathrm{M}$ and $\mathrm{N}$ the fused image be $\mathrm{L}$, then multi-scale decomposition representations can be given as $D_{m}, D_{n}$ and $D_{l}$ and the activity level measures can be denoted by $A_{m}$ and $A_{n}$. An expression for the measure of index of an multi-scale decomposition coefficient is given as $q=(v, x, y, z)$, where $v$ and $\mathrm{x}$ in particular designate the spatial position in a given frequency band of an image, while $k$ being the decomposition level and $l$ the frequency band of the decomposed images

Prior to choosing the selection scheme it is requisite to apply a grouping scheme[17] followed by a combining method to the multi-scale decomposition coefficients. Each coefficient has a set of corresponding coefficients in other frequency bands and decomposition level. Grouping schemes generally are discretized in three different categories, a no-grouping scheme, single-scale grouping and a multi-scale grouping scheme[17]. The preference of one over the other is a matter of the correspondence of the multi-scale decomposition coefficients with those of other frequency and decomposition levels. In case if the correspondence between multi-scale decomposition coefficients is ignored then such case becomes a no-grouping scheme case[17]. If the coefficients in decomposition levels are restricted to take same decision, then this is known as single-scale grouping[17]. While, if the coefficients of different levels or bands are given the liberty to take different decision then such a case becomes of multiscale grouping[17]. This paper encompasses the no-grouping scheme. Combining the source multi-scale decomposition coefficients to form a composite multi-scale decomposition representation, is done by using maximum selection rule. This selective scheme primarily invokes the most salient image features from among the source images. The justification for choosing selective scheme over averaging is the possible issue of pattern cancellation due to opposite contrast which remains unresolved despite the stabilized fused images.

In this paper, choose maximum scheme has been adopted. It implies that the coefficients with larger activity level has been picked up while discarding the other. It's a general practice to choose maximum only from the approximations that we obtain after decomposition. Conversely, in this paper, all the details along with the approximations are taken into consideration, i.e. select maximum rule is applied to all the decomposed bands and then the synthesis is done. The mathematical formula for an image $L$ can be described as $D_{l}(q)=D_{i}(q)$, where $i=M$ or $N$, depending on whichever source image satisfies the condition. If the coefficients of the decomposed band of either of image is equal, then weighted average of them is computed and is taken up by the fused image

$$
\begin{aligned}
& A_{i}(q)=\max \left(A_{m}(q), A_{n}(q)\right) \\
& A_{i}(q)=\frac{\left(A_{m}(q)+A_{n}(q)\right)}{2}
\end{aligned}
$$

A common procedure that follows the selection rule is consistency verification. It tries to take advantage of the possibility of computation of neighboring coefficients while calculating the composite multi-scale decomposition coefficient. As described earlier, maximum values of the activity measures are taken, signifying the presence of the dominant feature in the local area. Further, so as to record the selection results, a binary decision map of the size as that of the wavelet transform is generated. One possible scenario that might occur is that for an image, the dominant feature might be evident only in the center pixel, while for another image salient features might be from the pixels in the surrounding region of the center region of that window, in such cases the binary decision map is subjected to consistency verification[18]. Wherein, the binary decision map is filtered using a majority filter followed by negation and repeating the procedure once more. This selection scheme helps ensure that most of the salient features are subsumed into the fused image.

After having carried out the consistency verification step[18], the fused bands are then subjected to the process of synthesis as illustrated in the figure. 5. The fused approximations and the details along the vertical, horizontal and diagonal stretch are filtered along the columns and then along the rows along with up sampling, to obtain a final fused image.

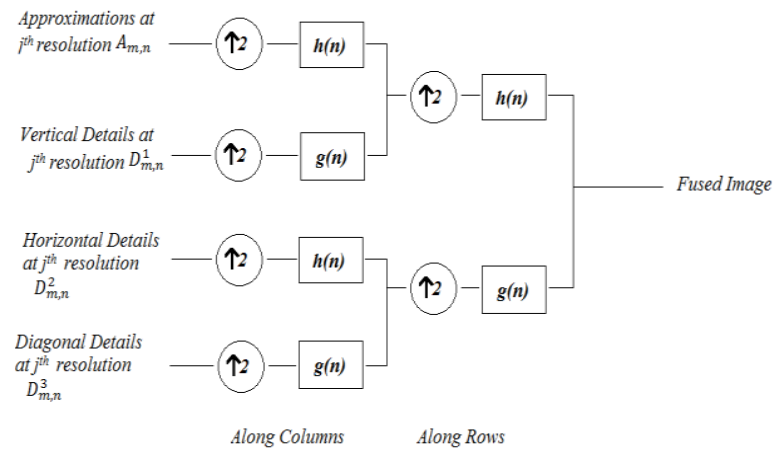

$h(n)$ - Low Pass Filter $g(n)$ - High Pass Filter

Fig 7: Synthesis process of an image 


\section{FUSION PERFORMANCE EVALUATION}

The concept of evaluation of fusion is more of a subjective criteria rather than objective. Since for objective evaluation it requires to have a solid ground truth documented that matches the application scenarios that fusion is meant to serve. The human perception probably has been by far the most commonly used criteria for subjective evaluation of image fusion. In recent years though, a number of methods have been proposed for the objective evaluation. MSE, Entropy, MI and PSNR are a few that have established a firm ground as common evaluation measures. Many more methods have been proposed, but none of them seem to have the advantages as that of the measures like PSNR and RMSE has to offer[18][19][20]. Although the human perception as a subjective measure is unparalleled by any of the objective methods, it is not something that has any scientific justification involved in it. Thus to have a scientific backing to the method the choice of objective evaluation is opted. This paper incorporates Entropy, PSNR and MSE as the objective criterion of evaluation of images.

\subsection{Entropy}

This measure is used to evaluate and describe the amount of information of the source images retained in the fused image. Each source image is referred to as a discrete random variable. Thus, the entropy of the image is given as follows.

$$
H(X)=-\sum_{x} p(x) \log p(x)
$$

In this paper, entropy of the fused images are calculated. With the help of this evaluation, the information content in the fused image can be known.

\subsection{MSE(Mean Squared Error)}

One of the most widely used and one of the simplest among the known quality metric is MSE. Apart from being simple in calculation it offers consistency in terms of optimization of mathematical calculation. The goal of this image fidelity measure is to compare two images by providing a quantitative score that describes the degree of similarity or conversely and/or the level of inconsistency between them. For the case of image fusion, MSE can be described as the sum of the mean squared error terms relative to both the source images. As the name itself implies, it is the mean over the total pixel number of the squared difference in the fused image pixel values and the source image pixel values. Though it has gained its popularity because of the advantage it offers, it lacks the ability to assess the image difference or better way to state it would be the similarity across different noise additions that are conducive hazards in fusion.

In this paper, the expression has been modified to meet the necessary conditions but only within the limits of its definition.

$$
\begin{aligned}
& M S E=\frac{\sum_{i=1}^{M} \sum_{j=1}^{N}(F(i, j)-A(i, j))^{2}}{M \times N} \\
&+\frac{\sum_{i=1}^{M} \sum_{j=1}^{N}(F(i, j)-B(i, j))^{2}}{M \times N}
\end{aligned}
$$

\subsection{PSNR}

PSNR is another image fusion quality measure that has been used in this paper. It involves direct use of the MSE value calculated, but offers a dissimilar informative details about the image fusion method, more specifically for this paper, provides information about the best wavelet basis for fusion purposes.

$$
P S N R=10 \log _{10}(255 / M S E)
$$

\section{EXPERIMENTAL VALIDATION}

The simulation of the whole process has been carried out on MATLAB. A Graphical User Interface has been constructed that invokes two images to be fused and gives a fused image as a result, along with a drop down menu to choose the different wavelet basis with which the images then undergo the whole fusion process. The results obtained from the simulation are published in this section. The images used are of 256x256 resolution. These images are grey scale images obtained from the MRI and CT scan of different clinical conditions of brain.

The following all figures belong to a particular clinical condition. The results discussed in table no. 1 are all taken in reference to images in the figure no. 8 which are MRI and CT scan images of the brain having a particular condition.

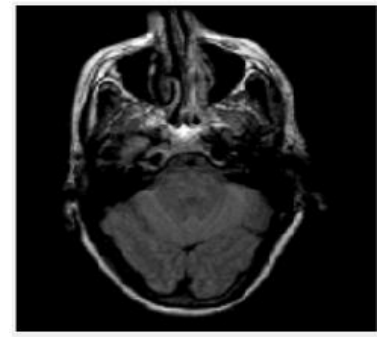

(a)

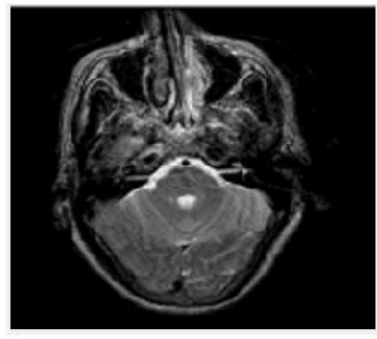

(b)
Fig 8: (a) MRI Image (b) CT Scan Image

The above two images are the source images obtained from MRI and CT scan of a certain clinical condition of brain. Firstly, these images are subjected to decomposition using different wavelets. The following images are displayed in the figure for illustration purpose. The wavelet used in their decomposition is Symlet $(\mathrm{N}=4)$.

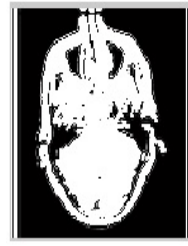

(a)

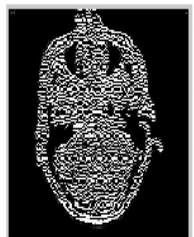

(b)

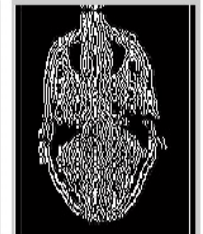

(c)

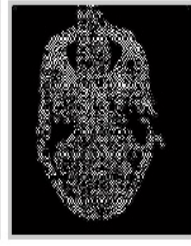

(d)
Fig 9: (a)Approximations (b) Horizontal Details (c) Vertical Details (d) Diagonal Details of MRI Image

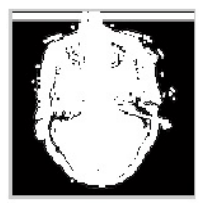

(a)

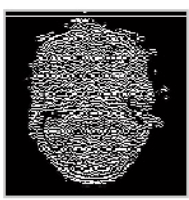

(b)

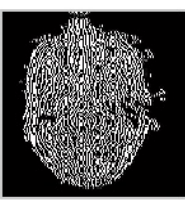

(c)

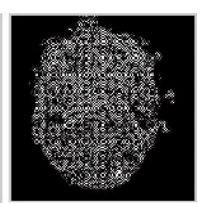

(d)
Fig 10: (a)Approximations (b) Horizontal Details (c) Vertical Details (d) Diagonal Details of CT Scan Image

These set of images are fused using the proposed fusion scheme. The resulting set of images that are obtained are as follows. 


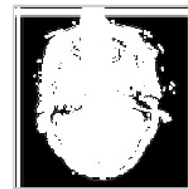

(a)

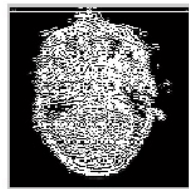

(b)

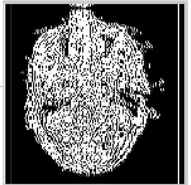

(c)

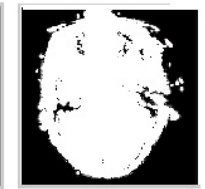

(d)
Fig 11: (a)Approximations (b) Horizontal Details (c) Vertical Details (d) Diagonal Details of fused images

These Images are later subjected to Inverse Discrete Wavelet Transformation. After having undergone IDWT a single fused image is obtained. The process is also better known as synthesis is performed. These set of source images are analyzed and synthesized using different wavelets as mentioned earlier. The MSE, PSNR and Entropy of fused images are obtained as result of the simulation. The observed results are presented in a tabular form in Table no. 1.

Table. 1: Results corresponding to the images displayed in figure. no. 8

\begin{tabular}{|c|c|c|c|}
\hline $\begin{array}{c}\text { Types of } \\
\text { Wavelets }\end{array}$ & PSNR & MSE & Entropy \\
\hline Haar & 50.3872 & 0.5948 & 14.0323 \\
\hline Daubechies-4 & 47.3042 & 1.2096 & 23.6144 \\
\hline Daubechies-8 & 40.2233 & 6.1765 & 27.9065 \\
\hline Symlet-4 & 46.2066 & 1.5575 & 23.6278 \\
\hline Symlet-8 & 44.2738 & 2.4305 & 24.515 \\
\hline
\end{tabular}

The following figure comprises of resultant fused images produced using different wavelets.

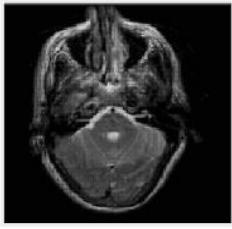

(a)

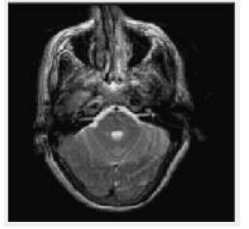

(b)

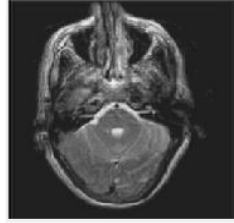

(c)

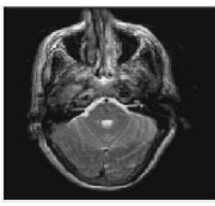

(d)

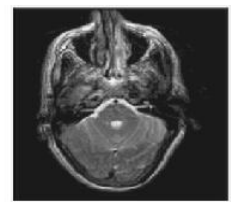

(e)
Fig 12: Fused using (a)Haar Wavelet (b) Daubechies - 8 (c) Daubechies - 16 (d) Symlet - 8 (e) Symlet - 16

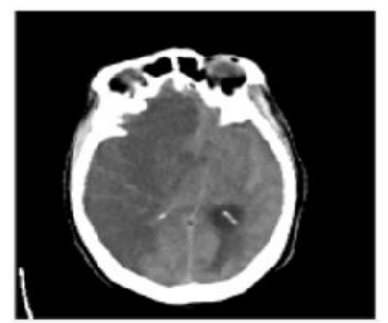

(a)

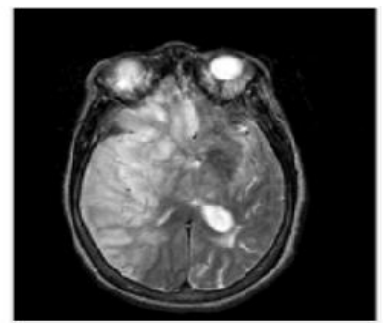

(b)
Fig 13: (a) MRI Image (b) CT Scan Image
Similarly, two more sets of MRI and CT scan images were used to obtain the results corresponding to them. The above figures and tables represent the results for the appropriate set of images. The results corresponding to the images in figure. no. 13. is presented in the Table. no. 2. Also, the resultant fused images are represented in figure. no. 14 .

Table. 2: Results corresponding to the images displayed in figure. no. 13

\begin{tabular}{|c|c|c|c|}
\hline $\begin{array}{c}\text { Types of } \\
\text { Wavelets }\end{array}$ & PSNR & MSE & Entropy \\
\hline Haar & 51.3327 & 0.4784 & 14.2365 \\
\hline Daubechies-4 & 50.3678 & 0.5974 & 24.6343 \\
\hline Daubechies-8 & 44.6801 & 2.2135 & 29.8842 \\
\hline Symlet-4 & 48.5046 & 0.9175 & 24.7057 \\
\hline Symlet-8 & 45.6069 & 1.7881 & 28.512 \\
\hline
\end{tabular}

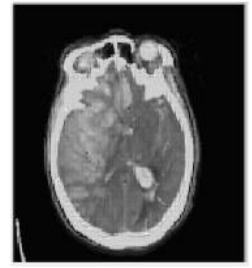

(a)

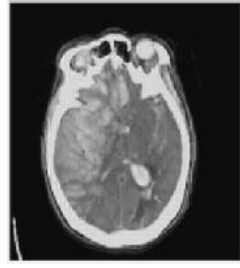

(b)

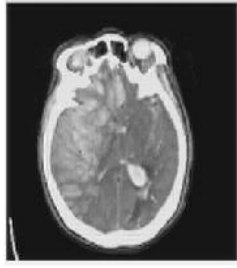

(c)

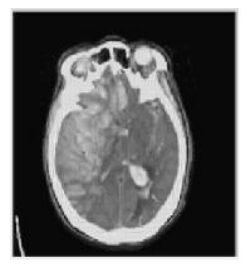

(d)

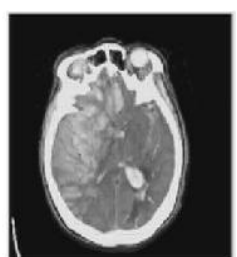

(e)
Fig 14: Fused using (a)Haar Wavelet (b) Daubechies - 8 (c) Daubechies - 16 (d) Symlet - 8 (e) Symlet - 16

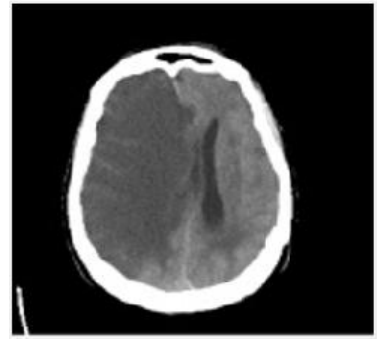

(a)

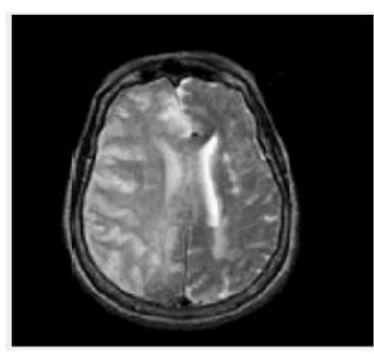

(b)
Fig 15: (a) MRI Image (b) CT Scan Image

The results corresponding to the above set of images are displayed in table. no. 3. in the adjacent column. The set of resultant fused images are collectively displayed in figure. no. 16.

Table. 3: Results corresponding to the images displayed in figure. no. 15

\begin{tabular}{|c|c|c|c|}
\hline $\begin{array}{c}\text { Types of } \\
\text { Wavelets }\end{array}$ & PSNR & MSE & Entropy \\
\hline Haar & 50.7130 & 0.5518 & 13.1810 \\
\hline Daubechies-4 & 50.1203 & 0.6325 & 22.053 \\
\hline Daubechies-8 & 47.8001 & 1.0791 & 26.493 \\
\hline
\end{tabular}




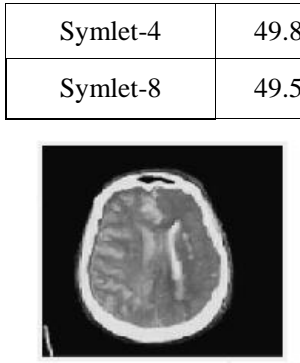

(a)

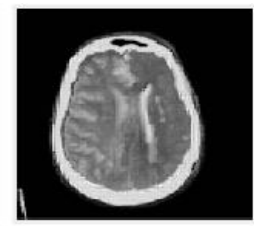

(b)

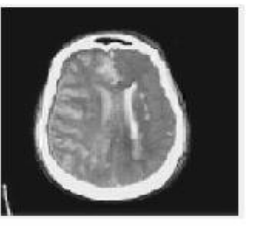

(c)

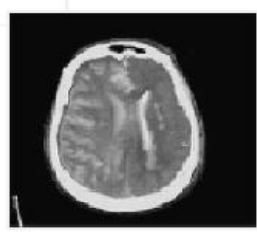

(d)

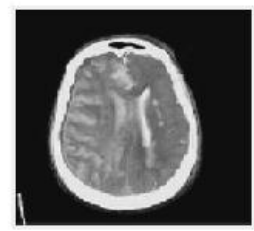

(e)
Fig 16: Fused using (a)Haar Wavelet (b) Daubechies - 8 (c) Daubechies - 16 (d) Symlet - 8 (e) Symlet - 16

\section{CONCLUSION}

This paper tries to draw a comparison between different wavelets from a perspective of fusion of medical images. From the obtained results it can be confirmed that the wavelets with lower order tend to give good results. Since the PSNR value corresponding to the lower values of order such as those for Daub4 is more as compared to Daub8. The same goes for Symlet wavelets. While the best results are obtained using the Haar wavelets, with highest PSNR value, least MSE and least Entropy. It can thus be conclude form above results that the wavelets that are higher in order shows a degradation in results of fusion when the aforementioned scheme of image fusion is adopted.

\section{ACKNOWLEDGMENT}

Corresponding author acknowledges Prof. Vicky Cheda, a faculty of Dept of Electronics \& Telecommunication, KJ Somaiya College Of Engineering, Vidyavihar(Mumbai) for providing insight and guidance in the experimental work in the laboratory.

\section{REFERENCES}

[1] Bushara N. Kayani, Anwar Majeed Mirza, Ajmal Bangash, Harron Iftikhar, "Pixel and Feature Level Multiresolution Image Fusion Based On Fuzzy Logic", in Innovations and Advanced Techniques in Computer and Information Sciences and Engineering, 2007, pp 129132.

[2] Shweta K. Shah, Prof. D. U. Shah. (2013, Mar.). Comparative Study of Image Fusion Techniques based on Spatial and Tranform Domain. International Journal of Innovative Research in Science, Engineering and Technology. [Online]. 3(3).

[3] Rohan Ashok Mandhare, Pragati Upadhyay, Sudha Gupta. (2013, June). Pixel Level Image Fusion Using Brovey Transform and Wavelet transform. International Journal of Advanced Research in Electrical, Electronics and Instrumentation Engineering. [Online]. 2(6). http://www.ijareeie.com/upload/june/25F_PIXEL.pdf.
[4] V. P. S. Naidu, J. R. Raol. (2008, May.). Pixel Level Image Fusion Using Wavelets and Principal Component Analysis

[5] Myungjin Choi, (2006, June) ,"A New Intensity-HueSaturation Fusion Approach to Image Fusion With a Tradeoff Parameter", IEEE Transactions on Geoscience and Remote Sensing, 44(6).

[6] H. Li, B. S. Manjunath, S. K. Mitra. "Multisensor image fusion using the wavelet transform", Graphical Models and Image Processing, 57(3), pp. 235- 245, 1995.

[7] Tianjiao Zeng, Renyi Hu, Yaodong He, and Yunqi Wang, "Image Fusion Using Laplacian Pyramid Transform," ECE Capstone Design Project, Rutgers School of Engineering, Spring 2014.

[8] A. Grossmann and J. Morlet, "Decomposition of Hardy functions into square integrable wavelets of constant shape," SIAM J. Math.. vol. 15. pp. 723-736. 1984

[9] Y. Meyer. "Ondelettes et foncttons splines," Srm. Equarions auxeril,ee.s Par-tie//es. Ecole Polytechnique. Paris, France, Dec. 1986.

[10] S. Mallat, A Wavelet Tour of Signal Processing, Academic Press,1999.

[11] Gonzalo Pajares, Jesus Manuel de la Cruz."A waveletbased image fusion tutorial", Pattern recognition. 37, Elsevier, pp, 1855-1872, 2004

[12] Haar A., "Zur Theorie der orthogonalen Funktionensysteme. Mathematische Annalen", 69, 331371.

[13] Porwik P., Lisowska A," The New Graphic Description of the Haar Wavelet Transform". Lecture Notes in Computer Science, Springer-Verlag, Berlin, Heidelberg, New York, 3039, 1-8.

[14] Zeng L., Jansen C. P., Marsch S., Unser M., Hunziker R,." Four- Dimensional Wavelet Compression of Arbitrarily Sized Echocardiographic Data", IEEE Transactions on Medical Imaging, 21(9), 1179-1188. 2003.

[15] Claypoole R., Davis G., Sweldens W., Baraniuk R. "Adaptive Wavelet Transforms for Image Coding" Asilomar Conference on Signals, Systems and Computers.

[16] Munoz A., Ertle R., Unser M."Continuous wavelet transform with arbitrary scales and $\mathrm{O}(\mathrm{N})$ complexity", Signal Processing, 82, 749-757.

[17] Z. Zhang, R.S. Blum, "A categorization of multi-scale decomposition based image fusion schemes with a performance study for a digital camera application", Proc. IEEE 87 (8) (1999) 1315-1326.

[18] J.-B. Martens and L. Meesters, "Image dissimilarity," Signal Processing, Vol. 70, pp. 155-176, Nov. 1998.

[19] VQEG, "Final report from the video quality experts group on the validation of objective models of video quality assessment," http://www.vqeg.org/, Mar. 2000.

[20] A. M. Eskicioglu and P.S. Fisher, "Image quality measures and their performance," IEEE Trans. Communications, vol. 34, pp. 2959-2965, Dec. 1995. 